\title{
WELL-POSEDNESS STUDY AND FINITE ELEMENT SIMULATION OF TIME-DOMAIN CYLINDRICAL AND ELLIPTICAL CLOAKS
}

\author{
JICHUN LI, YUNQING HUANG, AND WEI YANG
}

\begin{abstract}
The goal of this paper is to prove the well-posedness for the governing equations which are used for cylindrical cloaking simulation. A new time-domain finite element scheme is developed to solve the governing equations. Numerical results demonstrating the cloaking phenomenon with the cylindrical cloak are presented. We finally extend the analysis and simulation to an elliptical cloak model.
\end{abstract}

\section{INTRODUCTION}

The idea of invisibility cloaking using metamaterials got its start in 2006 when Pendry et al. [46] and Leonhardt [31 laid out the blueprints for making objects invisible to electromagnetic waves. In late 2006, a 2-D reduced cloak was successfully fabricated and demonstrated to work in the microwave frequency regime [48. This is the first practical realization of such a cloak, and the result matches well with the computer simulation [16] performed using the commercial package COMSOL. The basic principle of Pendry, Schurig, and Smith's cloaking is to use the form-invariant property of Maxwell's equations under coordinate transformation to define the permittivity and permeability of the cloaking metamaterial. We want to note that essentially the same idea was discussed earlier by Greenleaf, Lassas, and Uhlmann 20] for electrical impedance tomography. Of course, there exist many other promising schemes for achieving invisibility cloaks by using metamaterials. Examples include a scheme based on optical conformal mapping [31, one using anomalous localized resonance 41, one based on special but object-dependent coatings [1, and one using zero index metamaterials [24, 44, etc.

Since 2006, the study of using metamaterials to construct invisibility cloaks of different shapes (e.g. [28, 34, 45, 52]) has been a very hot research topic. Cloaks operating from microwave frequencies to optical frequencies have been investigated, more details and references on cloaking can be found in some recent reviews (e.g., 12, 21, 22, 51]) and a recent book by Leonhardt and Philbin [32. Numerical simulation [25,35] plays a very important role in modeling of invisibility cloaks and validating theoretical predictions. Generally speaking, cloaking simulation boils down

Received by the editor January 16, 2013 and, in revised form, July 22, 2013.

2010 Mathematics Subject Classification. Primary 78M10, 65N30, 65F10.

Key words and phrases. Maxwell's equations, invisibility cloak, finite element method, metamaterials.

The first author was supported by NSFC project 11271310 and NSF grant DMS-0810896.

The third author was supported by Hunan Education Department Key Project 10A117 and Hunan Provincial Innovation Foundation for Postgraduate (CX2011B243).

This work was supported in part by the NSFC Key Project 11031006 and IRT1179 of PCSIRT. 
to solving metamaterial Maxwell's equations in either frequency-domain or timedomain. Though there are many excellent publications on finite element analysis of Maxwell's equations (e.g., papers [7, 8, 10, 11, 13, 15, 18, 27, 47, 49, 50, 53, and books 17, 26, 35, 42 ), there has not been much mathematical analysis done for those cloaking models. In recent years, mathematicians have started investigating this fascinating subject, but most works are still limited to frequency-domain or the quasistatic regime by mainly solving the Helmholtz equation [2, 3, 5, 29, 30, 37, 39, 43, the conductivity problem [4, and the time-harmonic Maxwell's equations [6]. The advancement of broadband cloaks [23, 33, 40 makes time-domain cloaking simulation more appealing and necessary. The first experimental demonstration of a cloaking event in the time domain was present in 2012 [19. This achievement is a significant step towards the development of full spatio-temporal cloaking. In our very recent paper [36, we developed a time-domain finite element method to simulate a cylindrical cloak 52, and carried out some mathematical analysis for this model. However, due to the complexity of the modeling equations, the rigorous analysis of the well-posedness was unsuccessful then. One of the major contributions of this paper is to complete the well-posedness study of the cylindrical cloak. Also, we propose a more efficient finite element scheme than the one proposed in our previous work [36], because the new scheme only solves three unknowns instead of four.

The rest of the paper is organized as follows. We first describe the 2-D cylindrical cloak modeling equations in Section 2. Then in Section 3, we investigate the wellposedness of this model. Then we propose a new mixed finite element scheme for simulating the cylindrical cloak. Numerical results showing the cloaking phenomena obtained by the new scheme are illustrated in Section 4. In Section 5, we extend the well-posedness study to an elliptical cloak model [28, 45]. Numerical simulations of the elliptical cloak are also performed. We conclude the paper in Section 6 .

\section{The MODELING EQUATIONS}

The cloak modeling is based on the Faraday's Law and Ampere's Law written as follows:

$$
\begin{gathered}
\frac{\partial \boldsymbol{B}}{\partial t}=-\nabla \times \boldsymbol{E}, \\
\frac{\partial \boldsymbol{D}}{\partial t}=\nabla \times \boldsymbol{H},
\end{gathered}
$$

and the constitutive relations

$$
\boldsymbol{D}=\varepsilon \boldsymbol{E}, \quad \boldsymbol{B}=\mu \boldsymbol{H},
$$

where $\boldsymbol{E}$ and $\boldsymbol{H}$ are the electric and magnetic fields respectively, $\boldsymbol{D}$ and $\boldsymbol{B}$ are the electric displacement and magnetic induction respectively, $\varepsilon$ and $\mu$ are cloak permittivity and permeability, respectively. For the cylindrical cloak, the ideal material parameters in the polar coordinate system are given by [46]:

(4) $\varepsilon_{r}=\mu_{r}=\frac{r-R_{1}}{r}, \quad \varepsilon_{\phi}=\mu_{\phi}=\frac{r}{r-R_{1}}, \quad \varepsilon_{z}=\mu_{z}=\left(\frac{R_{2}}{R_{2}-R_{1}}\right)^{2} \frac{r-R_{1}}{r}$,

where $R_{1}$ and $R_{2}$ are the inner and outer radius of the cloak. In this case, $\boldsymbol{E}$ becomes a 2-D vector, and $\boldsymbol{H}$ is a scalar, i.e., we can write $\boldsymbol{E}=\left(E_{x}, E_{y}\right)^{\prime}$ and $H=H_{z}$, where the subindex $x, y$ or $z$ denotes the component in each direction. Moreover, 
we follow the convention to denote the 2-D curl operators: For a scalar function $H$, we define $\nabla \times H=\left(\frac{\partial H}{\partial y},-\frac{\partial H}{\partial x}\right)^{\prime}$; while for a vector function $\boldsymbol{E}=\left(E_{x}, E_{y}\right)^{\prime}$, we define $\nabla \times \boldsymbol{E}=\frac{\partial E_{y}}{\partial x}-\frac{\partial E_{x}}{\partial y}$.

Transforming the polar coordinate system to the Cartesian coordinate system, we can obtain [52:

$$
\varepsilon_{0} \varepsilon_{r} \varepsilon_{\phi} \boldsymbol{E}=\left[\begin{array}{cc}
\varepsilon_{r} \sin ^{2} \phi+\varepsilon_{\phi} \cos ^{2} \phi & \left(\varepsilon_{\phi}-\varepsilon_{r}\right) \sin \phi \cos \phi \\
\left(\varepsilon_{\phi}-\varepsilon_{r}\right) \sin \phi \cos \phi & \varepsilon_{r} \cos ^{2} \phi+\varepsilon_{\phi} \sin ^{2} \phi
\end{array}\right] \boldsymbol{D}
$$

Because the material parameters given in (4) cannot be used directly to simulate the time-domain cloak, we have to map the parameters using the dispersive medium models. Here we consider the Drude model for the permittivity:

$$
\varepsilon_{r}(\omega)=1-\frac{\omega_{p}^{2}}{\omega^{2}-j \omega \gamma},
$$

where $\gamma \geq 0$ and $\omega_{p}>0$ are the collision and plasma frequencies, respectively. Note that other dispersive material models (e.g., Debye, Lorentz etc. [35, pp. 232-235]) can also be considered here. Due to its simple form and easy implementation, the Drude model is the most widely used one in the modeling of metamaterials.

Substituting (6) into (5) and changing into time-domain, we have [52]:

$$
\varepsilon_{0} \varepsilon_{\phi}\left(\frac{\partial^{2}}{\partial t^{2}}+\gamma \frac{\partial}{\partial t}+w_{p}^{2}\right) \boldsymbol{E}=\left(\frac{\partial^{2}}{\partial t^{2}}+\gamma \frac{\partial}{\partial t}+w_{p}^{2}\right) M_{A} \boldsymbol{D}+\varepsilon_{\phi}\left(\frac{\partial^{2}}{\partial t^{2}}+\gamma \frac{\partial}{\partial t}\right) M_{B} \boldsymbol{D},
$$

where we denote $\boldsymbol{D}=\left(D_{x}, D_{y}\right)^{\prime}, I_{2}$ for the $2 \times 2$ identity matrix, and matrices

$$
M_{A}=\left[\begin{array}{cc}
\sin ^{2} \phi & -\sin \phi \cos \phi \\
-\sin \phi \cos \phi & \cos ^{2} \phi
\end{array}\right], M_{B}=\left[\begin{array}{cc}
\cos ^{2} \phi & \sin \phi \cos \phi \\
\sin \phi \cos \phi & \sin ^{2} \phi
\end{array}\right]=I_{2}-M_{A} .
$$

Similarly, we map the permeability using the Drude model [52]:

$$
\mu_{z}(\omega)=A\left(1-\frac{\omega_{p m}^{2}}{\omega^{2}-j \omega \gamma_{m}}\right)
$$

where $A=\frac{R_{2}}{R_{2}-R_{1}}$, and $\omega_{p m}>0$ and $\gamma_{m} \geq 0$ are the magnetic plasma and collision frequencies, respectively. Substituting (8) into (3), we obtain

$$
B_{z}=\mu_{o} \mu_{z} H_{z}=\mu_{0} A\left(1-\frac{\omega_{p m}^{2}}{\omega^{2}-j \omega \gamma_{m}}\right) H_{z},
$$

which in time-domain is equivalent to

$$
\left(\frac{\partial^{2}}{\partial t^{2}}+\gamma_{m} \frac{\partial}{\partial t}\right) B_{z}=\mu_{0} A\left(\frac{\partial^{2}}{\partial t^{2}}+\gamma_{m} \frac{\partial}{\partial t}+\omega_{p m}^{2}\right) H_{z}
$$

In summary, the time-domain cylindrical cloaking model is composed of equations (11), (2), (7), and (10), subject to proper boundary and initial conditions. To be more specific, inside the annulus $R_{1} \leq r \leq R_{2}$ (i.e., the so-called cloaking 
region), the governing equations are:

(11) $\frac{\partial \boldsymbol{B}}{\partial t}=-\nabla \times \boldsymbol{E}$,

(12) $\frac{\partial \boldsymbol{D}}{\partial t}=\nabla \times \boldsymbol{H}$

(13) $\varepsilon_{0} \varepsilon_{\phi}\left(\frac{\partial^{2}}{\partial t^{2}}+\gamma \frac{\partial}{\partial t}+w_{p}^{2}\right) \boldsymbol{E}=\left(\frac{\partial^{2}}{\partial t^{2}}+\gamma \frac{\partial}{\partial t}+w_{p}^{2}\right) M_{A} \boldsymbol{D}+\varepsilon_{\phi}\left(\frac{\partial^{2}}{\partial t^{2}}+\gamma \frac{\partial}{\partial t}\right) M_{B} \boldsymbol{D}$,

(14) $\left(\frac{\partial^{2}}{\partial t^{2}}+\gamma_{m} \frac{\partial}{\partial t}\right) B_{z}=\mu_{0} A\left(\frac{\partial^{2}}{\partial t^{2}}+\gamma_{m} \frac{\partial}{\partial t}+\omega_{p m}^{2}\right) H_{z}$.

We assume that the perfectly conducting (PEC) boundary condition is imposed on the inner cylindrical boundary so that any object can be cloaked inside, since no wave can be penetrated into the inner circle (i.e., the so-called cloaked region). Outside the cloaking region is the free space, which is governed by the standard Maxwell's equations in air. Note that when the physical parameters

$$
\varepsilon_{r}=\mu_{r}=1, \varepsilon_{\phi}=\mu_{\phi}=1, \varepsilon_{z}=\mu_{z}=1, \omega_{p}=\omega_{p m}=0,
$$

the constitutive equations (5) and (9) become as $\boldsymbol{D}=\varepsilon_{0} \boldsymbol{E}$ and $B_{Z}=\mu_{o} H_{z}$, respectively. In this case, (13) and (14) are redundant, while (11) and (12) just become the standard Maxwell's equations in air:

$$
\mu_{o} \frac{\partial \boldsymbol{H}}{\partial t}=-\nabla \times \boldsymbol{E}, \quad \varepsilon_{0} \frac{\partial \boldsymbol{E}}{\partial t}=\nabla \times \boldsymbol{H},
$$

i.e., the cylindrical cloaking model (11)-(14) is reduced to the simple Maxwell's equations in air. In the next section, we will study the well-posedness of this cloaking model.

\section{THE WELL-POSEDNESS OF THE CLOAKING MODEL}

To simplify the notation and analysis, in the rest of the paper we assume that

$$
\gamma=\gamma_{m}
$$

Multiplying (7) by $\mu_{0} A$, then differentiating the resultant with respect to $t$ and using (2), we have

$$
\begin{array}{rl}
\mu_{0} & A \varepsilon_{0} \varepsilon_{\phi}\left(\boldsymbol{E}_{t^{3}}+\gamma \boldsymbol{E}_{t^{2}}+\omega_{p}^{2} \boldsymbol{E}_{t}\right) \\
& =\mu_{0} A\left[\left(M_{A}+\varepsilon_{\phi} M_{B}\right)\left(D_{t^{3}}+\gamma D_{t^{2}}\right)+\omega_{p}^{2} M_{A} D_{t}\right] \\
& =\mu_{0} A\left(M_{A}+\varepsilon_{\phi} M_{B}\right) \nabla \times\left(H_{t^{2}}+\gamma H_{t}\right)+\mu_{0} A \omega_{p}^{2} M_{A} \nabla \times H .
\end{array}
$$

To simplify the notation, here and below we denote $H=H_{z}, M=M_{A}+\varepsilon_{\phi} M_{B}$, and the $k$-th order partial derivative of a function $u$ with respect to $t$ as $u_{t^{k}}=\frac{\partial^{k} u}{\partial t^{k}}$.

By (10) and (1), we have

$$
\mu_{0} A\left(H_{t^{2}}+\gamma H_{t}+\omega_{p m}^{2} H\right)=-\nabla \times \boldsymbol{E}_{t}-\gamma \nabla \times \boldsymbol{E} .
$$

Taking curl of (16) and substituting the resultant into the right-hand side of (15), we have

$$
\begin{gathered}
\mu_{0} \varepsilon_{0} A \varepsilon_{\phi}\left(\boldsymbol{E}_{t^{3}}+\gamma \boldsymbol{E}_{t^{2}}+\omega_{p}^{2} \boldsymbol{E}_{t}\right)+M \nabla \times \nabla \times \boldsymbol{E}_{t}+\gamma M \nabla \times \nabla \times \boldsymbol{E} \\
=-\mu_{0} A M \nabla \times\left(\omega_{p m}^{2} H\right)+\mu_{0} A \omega_{p}^{2} M_{A} \nabla \times H .
\end{gathered}
$$

First, we prove an interesting property of matrix $M$. 
Lemma 3.1. The matrix $M_{A}$ is symmetric and non-negative definite, and the matrix $M$ is symmetric positive definite.

Proof. By the definition of $M_{A}$, it is easy to see that for any vector $(u, v)^{\prime}$, we have

$$
\begin{aligned}
& (u, v) M_{A}\left(\begin{array}{l}
u \\
v
\end{array}\right)=\left(u^{2} \sin ^{2} \phi-2 u v \sin \phi \cos \phi+v^{2} \cos ^{2} \phi\right) \\
& =(u \sin \phi-v \cos \phi)^{2} \geq 0,
\end{aligned}
$$

which proves the non-negativeness of $M_{A}$.

Similarly, using the definitions of $M_{A}$ and $M_{B}$, and the fact that $\varepsilon_{\phi}>1$, we have

$$
\begin{aligned}
& (u, v)\left(M_{A}+\varepsilon_{\phi} M_{B}\right)\left(\begin{array}{c}
u \\
v
\end{array}\right) \\
& =\left(u^{2} \sin ^{2} \phi-2 u v \sin \phi \cos \phi+v^{2} \cos ^{2} \phi\right) \\
& \quad+\varepsilon_{\phi}\left(u^{2} \cos ^{2} \phi+2 u v \sin \phi \cos \phi+v^{2} \sin ^{2} \phi\right) \\
& =(u \sin \phi-v \cos \phi)^{2}+\varepsilon_{\phi}(u \cos \phi+v \sin \phi)^{2} \\
& >(u \sin \phi-v \cos \phi)^{2}+(u \cos \phi+v \sin \phi)^{2}=u^{2}+v^{2},
\end{aligned}
$$

which shows the positive definiteness of $M_{A}+\varepsilon_{\phi} M_{B}$.

By Lemma 3.1, the matrix $M$ is invertible, and its inverse is denoted as $M_{C}=$ $\left(M_{A}+\varepsilon_{\phi} M_{B}\right)^{-1}$. Moreover, we have

Lemma 3.2. For matrix $M_{C}=\left(M_{A}+\varepsilon_{\phi} M_{B}\right)^{-1}, M_{C} \cdot M_{A}=M_{A}$ holds true.

Proof. Note that

$$
\begin{aligned}
M_{A}+\varepsilon_{\phi} M_{B} & =I_{2}-M_{B}+\varepsilon_{\phi} M_{B}=I_{2}+\left(\varepsilon_{\phi}-1\right) M_{B}=I_{2}+\frac{R_{1}}{r-R_{1}} M_{B} \\
& =\left[\begin{array}{cc}
1+\frac{R_{1}}{r-R_{1}} \cos ^{2} \phi & \frac{R_{1}}{r-R_{1}} \sin \phi \cos \phi \\
\frac{R_{1}}{r-R_{1}} \sin \phi \cos \phi & 1+\frac{R_{1}}{r-R_{1}} \sin ^{2} \phi
\end{array}\right],
\end{aligned}
$$

from which we obtain its determinant $\operatorname{det}\left(M_{A}+\varepsilon_{\phi} M_{B}\right)=1+\frac{R_{1}}{r-R_{1}}=\frac{r}{r-R_{1}}$, and its inverse

$M_{C}=\frac{r-R_{1}}{r}\left[\begin{array}{cc}1+\frac{R_{1}}{r-R_{1}} \sin ^{2} \phi & -\frac{R_{1}}{r-R_{1}} \sin \phi \cos \phi \\ -\frac{R_{1}}{r-R_{1}} \sin \phi \cos \phi & 1+\frac{R_{1}}{r-R_{1}} \cos ^{2} \phi\end{array}\right]=\frac{r-R_{1}}{r}\left(I_{2}+\frac{R_{1}}{r-R_{1}} M_{A}\right)$.

Using the property $M_{A}^{2}=M_{A}$, we have

$$
M_{C} \cdot M_{A}=\frac{r-R_{1}}{r}\left(M_{A}+\frac{R_{1}}{r-R_{1}} M_{A}^{2}\right)=\frac{r-R_{1}}{r} \cdot \frac{r}{r-R_{1}} M_{A}=M_{A},
$$

which completes the proof.

To study the well-posedness, we will first rewrite the governing equations (16) and (17) into a weak formulation. The domain $\Omega$ is the annulus $R_{1}<r<R_{2}$, and the PEC boundary condition $\boldsymbol{n} \times \boldsymbol{E}=\mathbf{0}$ is imposed on $\partial \Omega$, where $\boldsymbol{n}$ denotes the unit outward normal to $\partial \Omega$.

Let us denote the spaces

$$
\begin{aligned}
& H(\operatorname{curl} ; \Omega)=\left\{\boldsymbol{v} \in\left(L^{2}(\Omega)\right)^{2} ; \nabla \times \boldsymbol{v} \in\left(L^{2}(\Omega)\right)^{2}\right\}, \\
& H_{0}(\operatorname{curl} ; \Omega)=\{\boldsymbol{v} \in H(\operatorname{curl} ; \Omega) ; \boldsymbol{n} \times \boldsymbol{v}=\mathbf{0} \text { on } \partial \Omega\} .
\end{aligned}
$$


Left multiplying (17) by $M_{C}$ and then by a test function $\phi \in H_{0}(\operatorname{curl} ; \Omega)$, and using integration by parts and similarly, multiplying (16) by a test function $\psi \in$ $L^{2}(\Omega)$, we obtain the following weak formulation: Find $\boldsymbol{E}, H$ such that

(i)

$$
\begin{aligned}
& \varepsilon_{0} \mu_{0} A\left[\left(\varepsilon_{\phi} M_{C} \boldsymbol{E}_{t^{3}}, \boldsymbol{\phi}\right)+\gamma\left(\varepsilon_{\phi} M_{C} \boldsymbol{E}_{t^{2}}, \boldsymbol{\phi}\right)+\left(\omega_{p}^{2} \varepsilon_{\phi} M_{C} \boldsymbol{E}_{t}, \boldsymbol{\phi}\right)\right] \\
& +\left(\nabla \times \boldsymbol{E}_{t}, \nabla \times \boldsymbol{\phi}\right)+\gamma(\nabla \times \boldsymbol{E}, \nabla \times \boldsymbol{\phi}) \\
& =-\mu_{0} A\left(\omega_{p m}^{2} H, \nabla \times \boldsymbol{\phi}\right)+\mu_{0} A\left(\omega_{p}^{2} M_{C} M_{A} \nabla \times H, \boldsymbol{\phi}\right), \quad \forall \boldsymbol{\phi} \in H_{0}(\operatorname{curl} ; \Omega),
\end{aligned}
$$

(ii)

$$
\begin{aligned}
& \mu_{0} A\left[\left(H_{t^{2}}, \psi\right)+\gamma\left(H_{t}, \psi\right)+\left(\omega_{p m}^{2} H, \psi\right)\right] \\
& =-\left(\nabla \times \boldsymbol{E}_{t}+\gamma \nabla \times \boldsymbol{E}, \psi\right) . \quad \forall \psi \in L^{2}(\Omega) .
\end{aligned}
$$

Below we first prove some bounds for the magnetic field $H$ in terms of the electric field $\boldsymbol{E}$.

Lemma 3.3. For any $t \in(0, T]$, we have

$$
\|\nabla \times H\|_{0}^{2}(t) \leq C\left[\|\nabla \times H\|_{0}^{2}(0)+\|\boldsymbol{D}\|_{0}^{2}(0)+\int_{0}^{t}\left(\left\|\boldsymbol{E}_{t^{2}}\right\|_{0}^{2}+\left\|\boldsymbol{E}_{t}\right\|_{0}^{2}+\|\boldsymbol{E}\|_{0}^{2}\right) d t\right] .
$$

Proof. Multiplying (7) by $M_{C}$ and using the fact $M_{C} \cdot M_{A}=M_{A}$, we obtain

$$
\boldsymbol{D}_{t^{2}}+\gamma \boldsymbol{D}_{t}+\omega_{p}^{2} M_{A} \boldsymbol{D}=\epsilon_{0} \epsilon_{\phi} M_{C}\left(\boldsymbol{E}_{t^{2}}+\gamma \boldsymbol{E}_{t}+\omega_{p}^{2} \boldsymbol{E}\right)
$$

Then multiplying this by $\boldsymbol{D}_{t}$ and integrating over $\Omega$, we have

$$
\frac{1}{2} \frac{d}{d t}\left[\left\|\boldsymbol{D}_{t}\right\|_{0}^{2}+\left(\omega_{p}^{2} M_{A} \boldsymbol{D}, \boldsymbol{D}\right)\right] \leq C\left(\left\|\boldsymbol{E}_{t^{2}}\right\|_{0}+\left\|\boldsymbol{E}_{t}\right\|_{0}+\|\boldsymbol{E}\|_{0}\right)\left\|\boldsymbol{D}_{t}\right\|_{0} .
$$

Integrating (24) from 0 to $t$, and using Cauchy-Schwarz inequality to the righthand side of (24), we have

$$
\begin{aligned}
& \frac{1}{2}\left[\left\|\boldsymbol{D}_{t}\right\|_{0}^{2}(t)+\left(\omega_{p}^{2} M_{A} \boldsymbol{D}, \boldsymbol{D}\right)(t)\right] \\
& \leq \frac{1}{2}\left[\left\|\boldsymbol{D}_{t}\right\|_{0}^{2}(0)+\left(\omega_{p}^{2} M_{A} \boldsymbol{D}, \boldsymbol{D}\right)(0)\right] \\
& \quad+\frac{1}{2} \int_{0}^{t}\left\|\boldsymbol{D}_{t}\right\|_{0}^{2} d t+C \int_{0}^{t}\left(\left\|\boldsymbol{E}_{t^{2}}\right\|_{0}^{2}+\left\|\boldsymbol{E}_{t}\right\|_{0}^{2}+\|\boldsymbol{E}\|_{0}^{2}\right) d t,
\end{aligned}
$$

from which, the fact $\boldsymbol{D}_{t}=\nabla \times H$, Lemma 3.1, and the Gronwall inequality, we conclude the proof.

Lemma 3.4. For any $t \in(0, T]$, we have

$$
\begin{aligned}
& \left\|H_{t}(t)\right\|_{0}^{2}+\left\|\omega_{p m} H(t)\right\|_{0}^{2} \\
& \quad \leq C\left[\left\|H_{t}(0)\right\|_{0}^{2}+\left\|\omega_{p m} H(0)\right\|_{0}^{2}+\int_{0}^{t}\left(\left\|\nabla \times \boldsymbol{E}_{t}\right\|_{0}^{2}+\gamma^{2}\|\nabla \times \boldsymbol{E}\|_{0}^{2}\right) d t\right] .
\end{aligned}
$$


Proof. Choosing $\psi=H_{t}$ in (23), and integrating from 0 to $t$, we have

$$
\begin{aligned}
& \frac{1}{2} \mu_{0} A\left(\left\|H_{t}(t)\right\|_{0}^{2}+\left\|\omega_{p m} H(t)\right\|_{0}^{2}\right) \\
& \leq \frac{1}{2} \mu_{0} A\left(\left\|H_{t}(0)\right\|_{0}^{2}+\left\|\omega_{p m} H(0)\right\|_{0}^{2}\right) \\
& \quad-\int_{0}^{t}\left(\nabla \times \boldsymbol{E}_{t}+\gamma \nabla \times \boldsymbol{E}, H_{t}\right) d t \\
& \leq \frac{1}{2} \mu_{0} A\left(\left\|H_{t}(0)\right\|_{0}^{2}+\left\|\omega_{p m} H(0)\right\|_{0}^{2}\right) \\
& \quad+\int_{0}^{t}\left(\frac{1}{2} \mu_{0} A\left\|H_{t}(t)\right\|_{0}^{2}+\frac{1}{2 \mu_{0} A}\left\|\nabla \times \boldsymbol{E}_{t}+\gamma \nabla \times \boldsymbol{E}\right\|_{0}^{2}\right) d t,
\end{aligned}
$$

together with the Gronwall inequality, we have, for any $t \in[0, T]$,

$$
\begin{aligned}
& \frac{1}{2} \mu_{0} A\left(\left\|H_{t}(t)\right\|_{0}^{2}+\left\|\omega_{p m} H(t)\right\|_{0}^{2}\right) \\
& \leq e^{T}\left[\frac{1}{2} \mu_{0} A\left(\left\|H_{t}(0)\right\|_{0}^{2}+\left\|\omega_{p m} H(0)\right\|_{0}^{2}\right)\right. \\
& \left.\quad+\frac{1}{\mu_{0} A} \int_{0}^{t}\left(\left\|\nabla \times \boldsymbol{E}_{t}\right\|_{0}^{2}+\gamma^{2}\|\nabla \times \boldsymbol{E}\|_{0}^{2}\right) d t\right],
\end{aligned}
$$

which completes the proof.

With the above preparation, now we can prove the following stability.

Theorem 3.1. For the solution of (22) and (23), the following stability holds true:

$$
\begin{aligned}
& \varepsilon_{0} \mu_{0} A\left[\left(\varepsilon_{\phi} M_{c} \boldsymbol{E}_{t^{2}}, \boldsymbol{E}_{t^{2}}\right)(t)+\left(\omega_{p}^{2} \varepsilon_{\phi} M_{c} \boldsymbol{E}_{t}, \boldsymbol{E}_{t}\right)(t)\right]+\left(\nabla \times \boldsymbol{E}_{t}, \nabla \times \boldsymbol{E}_{t}\right)(t) \\
& +(\nabla \times \boldsymbol{E}, \nabla \times \boldsymbol{E})(t)+A\left(\omega_{p}^{2} \varepsilon_{\phi} M_{c} \boldsymbol{E}, \boldsymbol{E}\right)(t) \\
& +\mu_{0} A\left(\left\|H_{t}\right\|_{0}^{2}+\left\|\omega_{p m} H\right\|_{0}^{2}\right)(t) \leq C F(0),
\end{aligned}
$$

where constant $C>0$, and the function $F(0)$ depends on initial conditions $\nabla \times$ $\boldsymbol{E}(0), \nabla \times \boldsymbol{E}_{t}(0), \boldsymbol{E}(0), \boldsymbol{E}_{t}(0), \boldsymbol{E}_{t^{2}}(0), H(0), \nabla \times H(0), H_{t}(0)$ and $\boldsymbol{D}(0)$.

Proof. Choosing $\phi=\boldsymbol{E}_{t^{2}}$ in (22), we obtain

$$
\begin{aligned}
& \frac{1}{2} \varepsilon_{0} \mu_{0} A \frac{d}{d t}\left[\left(\varepsilon_{\phi} M_{C} \boldsymbol{E}_{t^{2}}, \boldsymbol{E}_{t^{2}}\right)+\left(\omega_{p}^{2} \epsilon_{\phi} M_{C} \boldsymbol{E}_{t}, \boldsymbol{E}_{t}\right)\right]+\frac{1}{2} \frac{d}{d t}\left\|\nabla \times \boldsymbol{E}_{t}\right\|_{0}^{2} \\
& \leq-\gamma\left(\nabla \times \boldsymbol{E}, \nabla \times \boldsymbol{E}_{t^{2}}\right)-\mu_{0} A\left(\omega_{p m}^{2} H, \nabla \times \boldsymbol{E}_{t^{2}}\right)+\mu_{0} A\left(\omega_{p}^{2} M_{A} \nabla \times H, \boldsymbol{E}_{t^{2}}\right),
\end{aligned}
$$

and integrating from 0 to $t$, we have

$$
\begin{aligned}
& \frac{1}{2} \varepsilon_{0} \mu_{0} A\left[\left(\varepsilon_{\phi} M_{C} \boldsymbol{E}_{t^{2}}, \boldsymbol{E}_{t^{2}}\right)(t)+\left(\omega_{p}^{2} \epsilon_{\phi} M_{C} \boldsymbol{E}_{t}, \boldsymbol{E}_{t}\right)(t)\right]+\frac{1}{2}\left\|\nabla \times \boldsymbol{E}_{t}\right\|_{0}^{2}(t) \\
& \leq \frac{1}{2} \varepsilon_{0} \mu_{0} A\left[\left(\varepsilon_{\phi} M_{C} \boldsymbol{E}_{t^{2}}, \boldsymbol{E}_{t^{2}}\right)(0)+\left(\omega_{p}^{2} \epsilon_{\phi} M_{C} \boldsymbol{E}_{t}, \boldsymbol{E}_{t}\right)(0)\right]+\frac{1}{2}\left\|\nabla \times \boldsymbol{E}_{t}\right\|_{0}^{2}(0) \\
& -\gamma\left(\nabla \times \boldsymbol{E}, \nabla \times \boldsymbol{E}_{t}\right)(t)+\gamma\left(\nabla \times \boldsymbol{E}, \nabla \times \boldsymbol{E}_{t}\right)(0)+\gamma \int_{0}^{t}\left\|\nabla \times \boldsymbol{E}_{t}\right\|_{0}^{2} d t \\
& -\mu_{0} A\left(\omega_{p m}^{2} H, \nabla \times \boldsymbol{E}_{t}\right)(t)+\mu_{0} A\left(\omega_{p m}^{2} H, \nabla \times \boldsymbol{E}_{t}\right)(0) \\
& +\mu_{0} A \int_{0}^{t}\left(\omega_{p m}^{2} H_{t}, \nabla \times \boldsymbol{E}_{t}\right) d t+\mu_{0} A \int_{0}^{t}\left(\omega_{p}^{2} M_{A} \nabla \times H, \boldsymbol{E}_{t^{2}}\right) d t .
\end{aligned}
$$


Substituting the following estimates

(i) $\quad \gamma\left(\nabla \times \boldsymbol{E}, \nabla \times \boldsymbol{E}_{t}\right)(t) \leq \delta_{1}\left\|\nabla \times \boldsymbol{E}_{t}\right\|_{0}^{2}+\frac{\gamma^{2}}{4 \delta_{1}}\|\nabla \times \boldsymbol{E}\|_{0}^{2}$,

(ii) $\quad \mu_{0} A\left(\omega_{p m}^{2} H, \nabla \times \boldsymbol{E}_{t}\right)(t) \leq \delta_{2}\left\|\nabla \times \boldsymbol{E}_{t}\right\|_{0}^{2}+\frac{1}{4 \delta_{2}}\left(\mu_{0} A\right)^{2}\left\|\omega_{m p}^{2} H\right\|_{0}^{2}$,

(iii) $\quad \mu_{0} A \int_{0}^{t}\left(\omega_{p m}^{2} H_{t}, \nabla \times \boldsymbol{E}_{t}\right) d t \leq \frac{\mu_{0} A}{2} \int_{0}^{t}\left(\left\|H_{t}\right\|_{0}^{2}+\left\|\omega_{p m}^{2} \nabla \times \boldsymbol{E}_{t}\right\|_{0}^{2}\right) d t$,

(iv) $\quad \mu_{0} A \int_{0}^{t}\left(\omega_{p}^{2} M_{A} \nabla \times H, \boldsymbol{E}_{t^{2}}\right) d t \leq \frac{\mu_{0} A}{2} \int_{0}^{t}\left(\|\nabla \times H\|_{0}^{2}+\left\|\omega_{p}^{2} M_{A} \boldsymbol{E}_{t^{2}}\right\|_{0}^{2}\right) d t$, into (27), and denoting $r h s(0)$ for those terms at $t=0$, we obtain

$$
\begin{aligned}
& \frac{1}{2} \varepsilon_{0} \mu_{0} A\left[\left(\varepsilon_{\phi} M_{C} \boldsymbol{E}_{t^{2}}, \boldsymbol{E}_{t^{2}}\right)(t)+\left(\omega_{p}^{2} \epsilon_{\phi} M_{C} \boldsymbol{E}_{t}, \boldsymbol{E}_{t}\right)(t)\right] \\
& \quad+\left(\frac{1}{2}-\delta_{1}-\delta_{2}\right)\left\|\nabla \times \boldsymbol{E}_{t}\right\|_{0}^{2}(t) \\
& \leq \operatorname{rhs}(0)+\frac{\gamma^{2}}{4 \delta_{1}}\|\nabla \times \boldsymbol{E}\|_{0}^{2}+\gamma \int_{0}^{t}\left\|\nabla \times \boldsymbol{E}_{t}\right\|_{0}^{2} d t \\
& +\frac{1}{4 \delta_{2}}\left(\mu_{0} A\right)^{2}\left\|\omega_{p m}^{2} H\right\|_{0}^{2}+\frac{\mu_{0} A}{2} \int_{0}^{t}\left(\left\|H_{t}\right\|_{0}^{2}+\left\|\omega_{p m}^{2} \nabla \times \boldsymbol{E}_{t}\right\|_{0}^{2}\right) d t \\
& +\frac{\mu_{0} A}{2} \int_{0}^{t}\left(\|\nabla \times H\|_{0}^{2}+\left\|\omega_{p}^{2} M_{A} \boldsymbol{E}_{t^{2}}\right\|_{0}^{2}\right) d t .
\end{aligned}
$$

Similarly, choosing $\phi=\boldsymbol{E}$ in (22), we obtain

$$
\begin{aligned}
& \frac{1}{2} \frac{d}{d t}\left[\|\nabla \times \boldsymbol{E}\|_{0}^{2}+\left(\omega_{p}^{2} \epsilon_{\phi} M_{C} \boldsymbol{E}, \boldsymbol{E}\right)\right]+\gamma\|\nabla \times \boldsymbol{E}\|_{0}^{2} \\
& \quad=-\varepsilon_{0} \mu_{0} A\left[\left(\varepsilon_{\phi} M_{C} \boldsymbol{E}_{t^{3}}, \boldsymbol{E}\right)+\gamma\left(\epsilon_{\phi} M_{C} \boldsymbol{E}_{t^{2}}, \boldsymbol{E}\right)\right] \\
& \quad-\mu_{0} A\left(\omega_{p m}^{2} H, \nabla \times \boldsymbol{E}\right)+\mu_{0} A\left(\omega_{p}^{2} M_{A} \nabla \times H, \boldsymbol{E}\right),
\end{aligned}
$$

and integrating from 0 to $t$, we have

$$
\begin{aligned}
& \frac{1}{2}\left[\|\nabla \times \boldsymbol{E}\|_{0}^{2}(t)+\left(\omega_{p}^{2} \epsilon_{\phi} M_{C} \boldsymbol{E}, \boldsymbol{E}\right)(t)\right] \\
& \quad \leq \frac{1}{2}\left[\|\nabla \times \boldsymbol{E}\|_{0}^{2}(0)+\left(\omega_{p}^{2} \epsilon_{\phi} M_{C} \boldsymbol{E}, \boldsymbol{E}\right)(0)\right] \\
& \quad-\varepsilon_{0} \mu_{0} A\left(\varepsilon_{\phi} M_{C} \boldsymbol{E}_{t^{2}}, \boldsymbol{E}\right)(t)+\varepsilon_{0} \mu_{0} A\left(\varepsilon_{\phi} M_{C} \boldsymbol{E}_{t^{2}}, \boldsymbol{E}\right)(0) \\
& \quad+\varepsilon_{0} \mu_{0} A \int_{0}^{t}\left(\varepsilon_{\phi} M_{C} \boldsymbol{E}_{t^{2}}, \boldsymbol{E}_{t}\right) d t-\varepsilon_{0} \mu_{0} A \gamma \int_{0}^{t}\left(\epsilon_{\phi} M_{C} \boldsymbol{E}_{t^{2}}, \boldsymbol{E}\right) d t \\
& \quad-\mu_{0} A \int_{0}^{t}\left(\omega_{p m}^{2} H, \nabla \times \boldsymbol{E}\right) d t+\mu_{0} A \int_{0}^{t}\left(\omega_{p}^{2} M_{A} \nabla \times H, \boldsymbol{E}\right) d t .
\end{aligned}
$$

Let $\omega_{\min }>0$ be a constant such that $\omega_{p} \geq \omega_{\min }$, we have

$$
\begin{array}{rl}
\varepsilon_{0} \mu_{0} & A\left(\varepsilon_{\phi} M_{C} \boldsymbol{E}_{t^{2}}, \boldsymbol{E}\right)(t) \\
& \leq \epsilon_{0} \mu_{0} A\left[\delta_{4}\left(\epsilon_{\phi} M_{C} \boldsymbol{E}_{t^{2}}, \boldsymbol{E}_{t^{2}}\right)+\frac{1}{4 \delta_{4}}\left(\epsilon_{\phi} M_{C} \boldsymbol{E}, \boldsymbol{E}\right)\right] \\
& \leq \epsilon_{0} \mu_{0} A\left[\delta_{4}\left(\epsilon_{\phi} M_{C} \boldsymbol{E}_{t^{2}}, \boldsymbol{E}_{t^{2}}\right)+\frac{1}{4 \delta_{4} \omega_{\min }^{2}}\left(\omega_{p}^{2} \epsilon_{\phi} M_{C} \boldsymbol{E}, \boldsymbol{E}\right)\right] .
\end{array}
$$


Substituting (30) into (29), and multiplying the result by $6 \gamma^{2}$, we have

$$
\begin{aligned}
& 6 \gamma^{2}\left\{\frac{1}{2}\|\nabla \times \boldsymbol{E}\|_{0}^{2}(t)+\frac{1}{2}\left(1-\frac{\epsilon_{0} \mu_{0} A}{2 \delta_{4} \omega_{\min }^{2}}\right)\left(\omega_{p}^{2} \epsilon_{\phi} M_{C} \boldsymbol{E}, \boldsymbol{E}\right)(t)\right\} \\
& \leq 6 \gamma^{2}\left\{\frac{1}{2}\left[\|\nabla \times \boldsymbol{E}\|_{0}^{2}(0)+\left(\omega_{p}^{2} \epsilon_{\phi} M_{C} \boldsymbol{E}, \boldsymbol{E}\right)(0)\right]\right. \\
& \varepsilon_{0} \mu_{0} A \delta_{4}\left(\varepsilon_{\phi} M_{C} \boldsymbol{E}_{t^{2}}, \boldsymbol{E}_{t^{2}}\right)(t)+\varepsilon_{0} \mu_{0} A\left(\varepsilon_{\phi} M_{C} \boldsymbol{E}_{t^{2}}, \boldsymbol{E}\right)(0) \\
& +\varepsilon_{0} \mu_{0} A \int_{0}^{t}\left(\varepsilon_{\phi} M_{C} \boldsymbol{E}_{t^{2}}, \boldsymbol{E}_{t}\right) d t-\varepsilon_{0} \mu_{0} A \gamma \int_{0}^{t}\left(\epsilon_{\phi} M_{C} \boldsymbol{E}_{t^{2}}, \boldsymbol{E}\right) d t \\
& \left.\quad-\mu_{0} A \int_{0}^{t}\left(\omega_{p m}^{2} H, \nabla \times \boldsymbol{E}\right) d t+\mu_{0} A \int_{0}^{t}\left(\omega_{p}^{2} M_{A} \nabla \times H, \boldsymbol{E}\right) d t\right\} .
\end{aligned}
$$

Choosing $\delta_{1}=\delta_{2}=\frac{1}{8}$, and $\delta_{4}$ such that

$$
\frac{\epsilon_{0} \mu_{0} A}{\delta_{4} \omega_{\min }^{2}} \leq \frac{1}{2} \text { and } 6 \gamma^{2} \delta_{4}<\frac{1}{2}, \text { or } \frac{\epsilon_{0} \mu_{0} A}{\omega_{\min }^{2}} \leq \delta_{4}<\frac{1}{12 \gamma^{2}},
$$

then summing up (28) and (31), and using Lemmas 3.3 and 3.4 to bound those $H$ terms, we conclude the proof.

Remark 3.1. The first constraint in (32) is used to make sure that the second term on the left-hand side (LHS) of (31) is positive; while the second constraint in (32) is needed to guarantee that the third term on the right-hand side of (31) is less than the first term on the LHS of (28). When $\gamma=0$, we just drop the multiplier $6 \gamma^{2}$ in (31), and simply choose $\delta_{4}=\frac{1}{4}$ and $\delta_{1}=\delta_{2}=\frac{1}{8}$ to guarantee the stability (25).

Note that (32) always holds true in practice. For example, in cylindrical cloak simulation (e.g., [36, 38]), the following parameters are often chosen: $R_{1}=0.1 \mathrm{~m}$, $R_{2}=0.2 \mathrm{~m}, \omega=2 \pi f, \gamma=0.01 \omega$ with operating frequency $f=2 \mathrm{GHz}$. Using the fact that $1 / \sqrt{\epsilon_{0} \mu_{0}}=3 \cdot 10^{8} \mathrm{~m} / \mathrm{s}$, we can see that $\frac{\epsilon_{0} \mu_{0} A}{\omega_{\min }^{2}} \approx 1 /\left(2 * 9 * 10^{16} * \sqrt{2} * \pi * 2 *\right.$ $\left.10^{9}\right)=\frac{1}{36 \sqrt{2} \pi \cdot 10^{25}}$, which is much smaller than $\frac{1}{12 \gamma^{2}}=\frac{1}{12 *\left(0.01 * 2 \pi * 2 * 10^{9}\right)}=\frac{1}{192 \pi^{2} \cdot 10^{14}}$.

Finally, we can prove the existence and uniqueness of the solution for our cloaking model.

Theorem 3.2. For any $t \in[0, T]$, there exists a unique solution $(\boldsymbol{E}(\cdot, t), H(\cdot, t)) \in$ $H_{0}(\operatorname{curl} ; \Omega) \times H(\operatorname{curl} ; \Omega)$ of (22) and (23).

Proof. Taking the Laplace transform of (15), we obtain

$$
\begin{aligned}
& A \mu_{0} \epsilon_{0} \epsilon_{\phi}\left[s^{3} \hat{\boldsymbol{E}}-s^{2} \boldsymbol{E}_{0}-s \boldsymbol{E}_{0}^{\prime}-\boldsymbol{E}_{0}^{\prime \prime}+\gamma\left(s^{2} \hat{\boldsymbol{E}}-s \boldsymbol{E}_{0}-\boldsymbol{E}_{0}^{\prime}\right)+\omega_{p}^{2}\left(s \hat{\boldsymbol{E}}-\boldsymbol{E}_{0}\right)\right] \\
& =\mu_{0} A M \nabla \times\left[s^{2} \hat{H}-s H_{0}-H_{0}^{\prime}+\gamma\left(s \hat{H}-H_{0}\right)\right]+\mu_{0} A \omega_{p}^{2} M_{A} \nabla \times \hat{H}
\end{aligned}
$$

or

$$
\begin{aligned}
& A \mu_{0} \epsilon_{0} \epsilon_{\phi}\left(s^{3}+\gamma s^{2}+\omega_{p}^{2} s\right) \hat{\boldsymbol{E}} \\
& =\mu_{0} A M \nabla \times\left(s^{2} \hat{H}+\gamma s \hat{H}\right)+\mu_{0} A \omega_{p}^{2} M_{A} \nabla \times \hat{H}+F_{0}(s),
\end{aligned}
$$

where $F_{0}(s)$ denotes all the terms related to initial conditions. 
Similarly, taking the Laplace transform of (16), we obtain

$$
\mu_{0} A\left(s^{2} \hat{H}+\gamma s \hat{H}+\omega_{p m}^{2} \hat{H}\right)=-\nabla \times(s \hat{\boldsymbol{E}}+\gamma \hat{\boldsymbol{E}})+G_{0}(s),
$$

from which we obtain

$$
\hat{H}=\frac{1}{\mu_{0} A\left(s^{2}+\gamma s+\omega_{p m}^{2}\right)}\left[-\nabla \times(s+\gamma) \hat{\boldsymbol{E}}+G_{0}(s)\right],
$$

where $G_{0}(s)$ denotes all the terms related to initial conditions.

Substituting (34) into (33) to eliminate $\hat{H}$, we obtain

$$
\begin{aligned}
& A \mu_{0} \epsilon_{0} \epsilon_{\phi}\left(s^{3}+\gamma s^{2}+\omega_{p}^{2} s\right) \hat{\boldsymbol{E}}=-\mu_{0} A M \nabla \times\left[\frac{\left(s^{2}+\gamma s\right)}{\mu_{0} A\left(s^{2}+\gamma s+\omega_{p m}^{2}\right)}(s+\gamma) \nabla \times \hat{\boldsymbol{E}}+\widetilde{G}_{0}(s)\right] \\
& -\mu_{0} A \omega_{p}^{2} M_{A} \nabla \times\left[\frac{(s+\gamma)}{\mu_{0} A\left(s^{2}+\gamma s+\omega_{p m}^{2}\right)} \nabla \times \hat{\boldsymbol{E}}+\widetilde{G}_{0}(s)\right]+F_{0}(s)
\end{aligned}
$$

or

$$
\begin{aligned}
A \mu_{0} \epsilon_{0} \epsilon_{\phi} M_{C}\left(s^{3}+\gamma s^{2}+\omega_{p}^{2} s\right) \hat{\boldsymbol{E}} & +\nabla \times\left(\frac{s(s+\gamma)^{2}}{s^{2}+\gamma s+\omega_{p m}^{2}} \nabla \times \hat{\boldsymbol{E}}\right) \\
& +\omega_{p}^{2} M_{A} \nabla \times\left(\frac{(s+\gamma)}{s^{2}+\gamma s+\omega_{p m}^{2}} \nabla \times \hat{\boldsymbol{E}}\right)=\widetilde{F}_{0}(s) .
\end{aligned}
$$

Note that the matrix $s(s+\gamma) I_{2}+\omega_{p}^{2} M_{A}$ is symmetric positive definite, and we denote its inverse $M_{D}=\left[s(s+\gamma) I_{2}+\omega_{p}^{2} M_{A}\right]^{-1}$. Moreover, we denote $p(s)=$ $s\left(s^{2}+\gamma s+\omega_{p}^{2}\right)\left(s^{2}+\gamma s+\omega_{p m}^{2}\right)$, and rewrite (35) as

$$
A \mu_{0} \epsilon_{0} \epsilon_{\phi} p(s) M_{D} M_{C} \hat{\boldsymbol{E}}+\nabla \times((s+\gamma) \nabla \times \hat{\boldsymbol{E}})=F_{0}^{*}(s) .
$$

The weak formulation of (36): Find $\hat{\boldsymbol{E}} \in H_{0}(\operatorname{curl} ; \Omega)$ such that

$$
A \mu_{0} \epsilon_{0}\left(\epsilon_{\phi} p(s) M_{D} M_{C} \hat{\boldsymbol{E}}, \phi\right)+((s+\gamma) \nabla \times \hat{\boldsymbol{E}}, \nabla \times \phi)=\left(F_{0}^{*}(s), \phi\right),
$$

holds true for any $\phi \in H_{0}(\operatorname{curl} ; \Omega)$. The existence of a unique solution $\hat{\boldsymbol{E}} \in$ $H_{0}(\operatorname{curl} ; \Omega)$ of (37) is guaranteed by the Lax-Milgram lemma if the matrix $M_{D} M_{C}$ is symmetric positive definite, which will be proved below.

After some algebraic calculation, we obtain

$$
M_{D}=\frac{1}{\operatorname{det}}\left[s(s+\gamma) I_{2}+\omega_{p}^{2} M_{B}\right]
$$

where det $=s^{2}(s+\gamma)^{2}+\omega_{p}^{2} s(s+\gamma)$. Hence we have

$$
\begin{aligned}
M_{D} M_{C} & =\frac{1}{\operatorname{det}}\left[s(s+\gamma) I_{2}+\omega_{p}^{2} M_{B}\right] \cdot \frac{r-R_{1}}{r}\left(I_{2}+\frac{R_{1}}{r-R_{1}} M_{A}\right) \\
& =\frac{1}{\operatorname{det}} \cdot \frac{r-R_{1}}{r} \cdot\left\{s(s+\gamma) I_{2}+\omega_{p}^{2} M_{B}+\frac{s(s+\gamma) R_{1}}{r-R_{1}} M_{A}+\omega_{p}^{2} \cdot \frac{R_{1}}{r-R_{1}} M_{B} M_{A}\right\} \\
& =\frac{1}{\operatorname{det}} \cdot \frac{r-R_{1}}{r} \cdot\left\{s(s+\gamma) I_{2}+\omega_{p}^{2} M_{B}+\frac{s(s+\gamma) R_{1}}{r-R_{1}} M_{A}\right\},
\end{aligned}
$$

where in the last step we used the fact that $M_{B} M_{A}=\left(I_{2}-M_{A}\right) M_{A}=0$. 


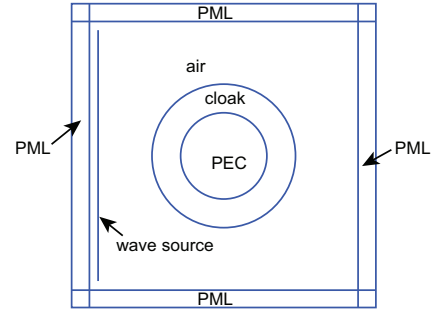

Figure 1. (a): The cloaking setup; (b): An exemplary coarse mesh.

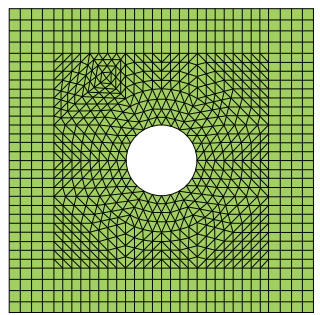

Using the facts that for any nonzero vector $(u, v)^{\prime}$,

$$
(u, v) M_{A}\left(\begin{array}{l}
u \\
v
\end{array}\right) \geq 0, \quad(u, v) M_{B}\left(\begin{array}{l}
u \\
v
\end{array}\right) \geq 0,
$$

we see that $(u, v) M_{D} M_{C}\left(\begin{array}{l}u \\ v\end{array}\right)>0$, which shows that the matrix $M_{D} M_{C}$ is symmetric positive definite.

The existence and uniqueness of $\hat{H}$ is implied from the existence and uniqueness of $\hat{\boldsymbol{E}}$ and (34). It is easy to see that (34) implies $\hat{H} \in L^{2}(\Omega)$. Furthermore, through some algebraic calculation, we see that (33) is equivalent to

$$
\nabla \times \hat{H}=\epsilon_{0} \epsilon_{\phi}\left(s^{3}+\gamma s^{2}+\omega_{p}^{2} s\right) M_{D} M_{C} \hat{\boldsymbol{E}}-\frac{1}{\mu_{0} A} M_{D} M_{C} F_{0}(s),
$$

which implies that $\nabla \times \hat{H} \in L^{2}(\Omega)$. Hence $\hat{H} \in H(\operatorname{curl} ; \Omega)$. The inverse Laplace transforms of functions $\hat{\boldsymbol{E}}$ and $\hat{H}$ are solutions of the time-dependent problem (22) and (23). This completes the proof.

\section{NumericAl RESUlts}

In this section we present some cloaking simulations obtained with the cylindrical cloaking model. Here we design a more efficient mixed finite element method than that proposed in our previous work [36].

To solve our problem efficiently, we partition the physical domain $\Omega$ by a family of regular meshes $T^{h}$ with hybrid grids: triangles in both cloaking and free space regions; rectangles in the perfectly matched layer (PML) region. The PML concept was introduced by Berenger [9] in 1994 to simulate wave propagation problems with open boundaries. Since 1994 there have been many investigations of PML for both Maxwell's equations and other wave equations. We do not repeat the details about how to use PML for the cylindrical cloaking simulation, which were discussed in our previous work 36]. The specific cloaking simulation setup and a sample mesh are given in Figure 1

Denote $\tau$ for the time step size. With notations

$$
\begin{gathered}
\delta_{\tau} u^{n+\frac{1}{2}}=\frac{u^{n+\frac{1}{2}}-u^{n-\frac{1}{2}}}{\tau}, \quad \delta_{\tau}^{2} u^{n+\frac{1}{2}}=\frac{\delta_{\tau} u^{n+\frac{1}{2}}-\delta_{\tau} u^{n-\frac{1}{2}}}{\tau}, \\
\check{u}^{n+\frac{1}{2}}=\frac{1}{2 \tau}\left(3 u^{n+\frac{1}{2}}-4 u^{n-\frac{1}{2}}+u^{n-\frac{3}{2}}\right), \quad \hat{u}^{n}=\frac{u^{n+\frac{1}{2}}+2 u^{n-\frac{1}{2}}+u^{n-\frac{3}{2}}}{4},
\end{gathered}
$$


we can construct a leap-frog mixed finite element scheme for solving our cloaking model: for $n=1,2, \ldots$, find $\boldsymbol{D}_{h}^{n+\frac{1}{2}}, \boldsymbol{E}_{h}^{n+\frac{1}{2}} \in V_{h}^{0}, H_{h}^{n} \in U_{h}$ such that

$$
\begin{aligned}
& \left(\delta_{\tau} \boldsymbol{D}_{h}^{n+\frac{1}{2}}, \phi_{h}\right)=\left(H_{h}^{n}, \nabla \times \phi_{h}\right) \\
& \varepsilon_{0}\left(\varepsilon_{\phi} \delta_{\tau}^{2} \boldsymbol{E}_{h}^{n+\frac{1}{2}}, \varphi_{h}\right)+\varepsilon_{0} \gamma\left(\varepsilon_{\phi} \check{\boldsymbol{E}}_{h}^{n+\frac{1}{2}}, \varphi_{h}\right)+\varepsilon_{0}\left(\omega_{p}^{2} \varepsilon_{\phi} \hat{\boldsymbol{E}}_{h}^{n}, \varphi_{h}\right) \\
& \quad=\left(M \delta_{\tau}^{2} \boldsymbol{D}_{h}^{n+\frac{1}{2}}, \varphi_{h}\right)+\gamma\left(M \check{\boldsymbol{D}}_{h}^{n+\frac{1}{2}}, \varphi_{h}\right)+\left(\omega_{p}^{2} M_{A} \hat{\boldsymbol{D}}_{h}^{n}, \varphi_{h}\right), \\
& \mu_{0} A\left(\left(\delta_{\tau}^{2} H_{h}^{n+1}, \psi_{h}\right)+\gamma\left(\check{H}_{h}^{n+1}, \psi_{h}\right)+\left(\omega_{p}^{2} \hat{H}_{h}^{n+\frac{1}{2}}, \psi_{h}\right)\right) \\
& \quad=-\left(\nabla \times \check{\boldsymbol{E}}_{h}^{n+\frac{1}{2}}+\gamma \nabla \times \hat{\boldsymbol{E}}_{h}^{n}, \psi_{h}\right)
\end{aligned}
$$

hold true for any $\phi_{h}, \varphi_{h} \in V_{h}^{0}, \psi_{h} \in U_{h}$. Recall that $M=M_{A}+\varepsilon_{\phi} M_{B}$. Here the mixed finite element spaces $\boldsymbol{U}_{h}$ and $\boldsymbol{V}_{h}$ are chosen as 35, 42. for rectangular elements $K \in T^{h}$,

$$
\begin{aligned}
& \boldsymbol{U}_{h}=\left\{\psi_{h} \in L^{2}(\Omega):\left.\psi_{h}\right|_{K} \in Q_{0,0}, \forall K \in T^{h}\right\}, \\
& \boldsymbol{V}_{h}=\left\{\phi_{h} \in H(\operatorname{curl} ; \Omega):\left.\phi_{h}\right|_{K} \in Q_{0,1} \times Q_{1,0}, \forall K \in T^{h}\right\},
\end{aligned}
$$

where $Q_{i, j}$ denotes the space of polynomials whose degrees are less than or equal to $i$ and $j$ in variables $x$ and $y$, respectively. While on triangular elements, we choose

$$
\begin{aligned}
& \boldsymbol{U}_{h}=\left\{\psi_{h} \in L^{2}(\Omega):\left.\psi_{h}\right|_{K} \text { is a piecewise constant, } \forall K \in T^{h}\right\}, \\
& \boldsymbol{V}_{h}=\left\{\phi_{h} \in H(\operatorname{curl} ; \Omega):\left.\phi_{h}\right|_{K}=\operatorname{span}\left\{\lambda_{i} \nabla \lambda_{j}-\lambda_{j} \nabla \lambda_{i}\right\}, i, j=1,2,3, \forall K \in T^{h}\right\},
\end{aligned}
$$

where $\lambda_{i}$ denotes the standard linear basis function at vertex $i$ of element $K$. The space

$$
\boldsymbol{V}_{h}^{0}=\left\{\phi_{h} \in \boldsymbol{V}_{h}, \boldsymbol{n} \times \phi_{h}=\mathbf{0} \text { on } \partial \Omega\right\}
$$

is introduced to impose the perfect conducting boundary condition $\boldsymbol{n} \times \boldsymbol{E}=\mathbf{0}$.

Note that the scheme (39)-41] is very easy to solve. At each time step, we first solve (39) for $\boldsymbol{D}_{h}^{n+\frac{1}{2}}$, then solve (40) for $\boldsymbol{E}_{h}^{n+\frac{1}{2}}$, followed by $H_{h}^{n+1}$ from (41). Compared to the scheme proposed in our previous work [36], the current one is more efficient since we only solve for three unknowns instead of four in 36.

In our simulation we choose $R_{1}=0.1, R_{2}=0.2$, the operating frequency $f=2.0$ $\mathrm{GHz}$, and the lossless case with $\gamma=\gamma_{m}=0$. The real mesh used is one obtained by uniformly refining the coarse mesh shown in Figure 1 three times, which contains 65536 triangular elements and 28672 rectangular elements. The corresponding time step $\tau=2 \cdot 10^{-13}$ second, i.e., 0.2 picosecond (ps), is chosen to guarantee the stability of our explicit leap-frog explicit scheme. 

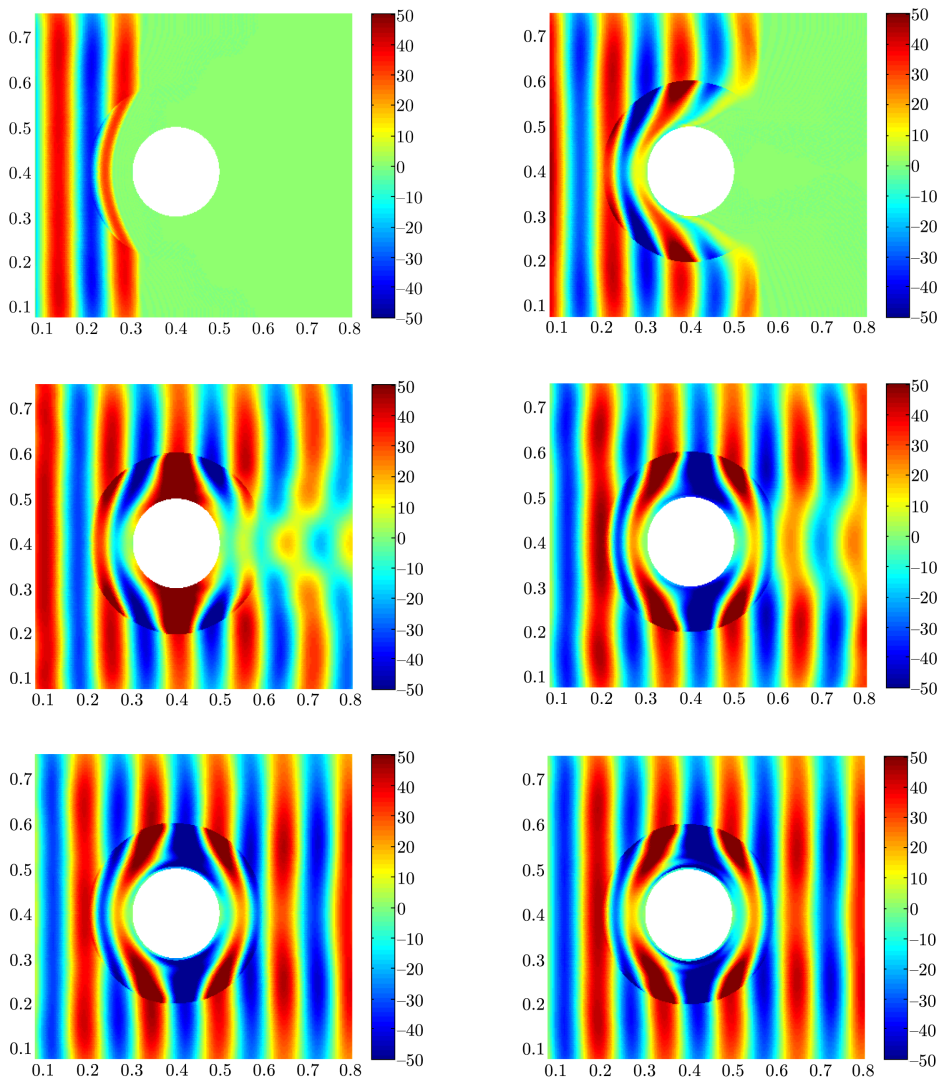

Figure 2. The $E_{y}$ fields at various times: (a) $t=0.8 n s(4,000$ steps); (b) $t=1.6 \mathrm{~ns}$ (8,000 steps); (c) $t=3.2 \mathrm{~ns}$ (16,000 steps); (d) $t=4 n s$ (20,000 steps); (e) $t=6 n s$ (30,000 steps); (f) $t=8 n s$ (40,000 steps).

Below we present two examples. The first one is used to make sure that our new scheme can produce the same result as our old scheme [36. The second one is a cloaking simulation obtained with an incident wave generated by a point source.

Example 1. The incident wave is generated by a plane wave $H_{z}=0.1 \sin (\omega t)$ with $\omega=2 \pi f$. The obtained electric fields $E_{y}$ at different time steps are presented in Figure 2, which is consistent as our previous work.

Example 2. In this example, the incident wave is generated by a point source $H_{z}=0.1 \sin (\omega t)$ imposed at a point $(0.078,0.4)$. The electric fields $E_{y}$ at different time steps are presented in Figure 3. which shows clearly that the incident wave after passing the cloaking region resumes the wave pattern in free space. Hence any object placed inside the cloak is undetected by the external electromagnetic waves. 

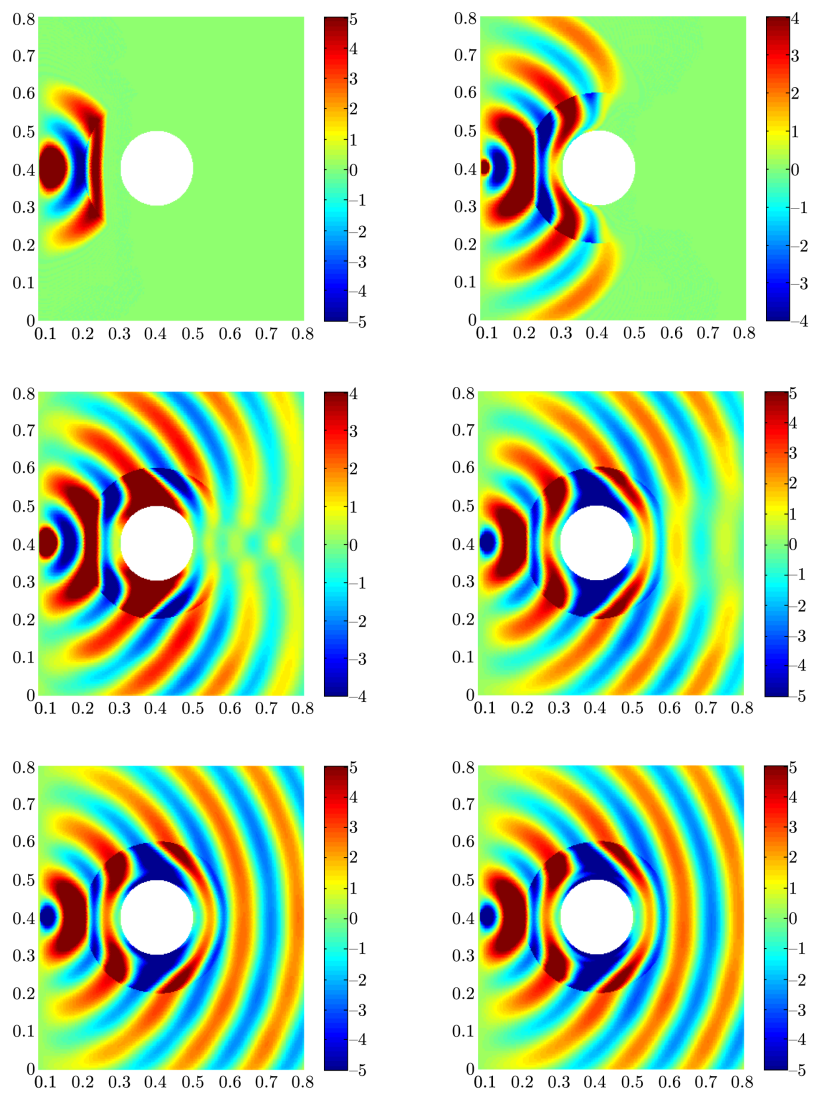

Figure 3 . The $E_{y}$ fields at various times: (a) $t=0.8 n s ;$ (b) $t=1.6 n s ;$ (c) $t=3.2 n s$; (d) $t=4 n s ;$ (e) $t=6 n s$; (f) $t=8 n s$.

\section{Extension to an Elliptical CloAK}

5.1. The governing equations. Based on the idea of transformation optics 46] (see also [35]), an elliptical cloak can be similarly designed [28. Consider an elliptical shell with semi-axes $a$ and $b$ in the $y$ direction; and semi-axes $k a$ and $k b$ in the $x$ direction; cf. Figure 4 . Here $k$ denotes the axis ratio, and gives different cloaks: a horizontal elliptical cloak when $k>1$, a vertical elliptical cloak when $k<1$ and a circular cloak when $k=1$.

In the elliptical cloak region (described by $k a<r=\sqrt{x^{2}+k^{2} y^{2}}<k b$ ), the relative permittivity and permeability can be expressed in the Cartesian coordinate system as:

$$
\epsilon=\mu=\left(\begin{array}{ccc}
\epsilon_{x x} & \epsilon_{x y} & 0 \\
\epsilon_{x y} & \epsilon_{y y} & 0 \\
0 & 0 & \epsilon_{z z}
\end{array}\right)
$$



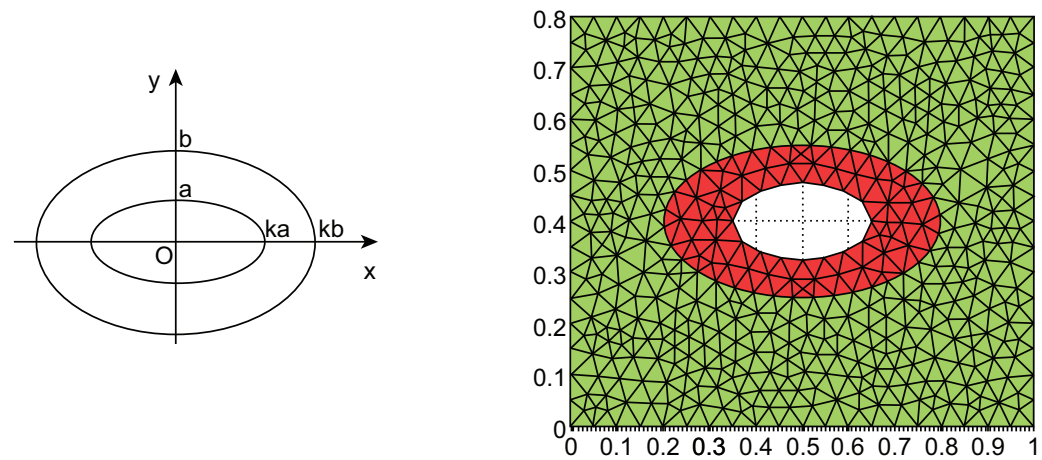

Figure 4. (Left) An elliptical cloak in the Cartesian coordinate system; (Right) An exemplary coarse mesh.

where

$$
\begin{aligned}
\epsilon_{x x} & =\frac{r}{r-k a}+\frac{k^{2} a^{2} R^{2}-2 k a r^{3}}{(r-k a) r^{5}} x^{2}, \\
\epsilon_{x y} & =\frac{k^{2} a^{2} R^{2}-k a\left(1+k^{2}\right) r^{3}}{(r-k a) r^{5}} x y, \\
\epsilon_{y y} & =\frac{r}{r-k a}+\frac{k^{2} a^{2} R^{2}-2 k^{3} a r^{3}}{(r-k a) r^{5}} y^{2}, \\
\epsilon_{z z} & =\left(\frac{b}{b-a}\right)^{2} \frac{r-k a}{r} .
\end{aligned}
$$

Here $R=\sqrt{x^{2}+k^{4} y^{2}}$. Detailed derivation can be found in [28].

Since $\epsilon$ is symmetric, it can be diagonalized by the eigenvalue matrix $\Lambda$ and a corresponding orthogonal matrix $P$, i.e., $\epsilon=P \Lambda P^{T}$, where [45]:

$$
\Lambda=\left(\begin{array}{ccc}
\lambda_{1} & 0 & 0 \\
0 & \lambda_{2} & 0 \\
0 & 0 & \lambda_{3}
\end{array}\right), \quad P=\left(\begin{array}{ccc}
p_{1} & p_{2} & 0 \\
-p_{2} & p_{1} & 0 \\
0 & 0 & 1
\end{array}\right)
$$

Here we denote

$$
\begin{aligned}
& \lambda_{1}=\frac{m-1}{m+1}, \quad \lambda_{2}=\frac{1}{\lambda_{1}}, \quad \lambda_{3}=\epsilon_{z z} \\
& m=\sqrt{1+\frac{4 r^{5}(r-k a)}{k^{2} a^{2} R^{2}\left(x^{2}+y^{2}\right)}}, \quad p_{1}=\frac{\epsilon_{x y}}{\sqrt{\epsilon_{x y}^{2}+\left(\lambda_{2}-\epsilon_{y y}\right)^{2}}}, \quad p_{2}=\frac{\lambda_{2}-\epsilon_{y y}}{\sqrt{\epsilon_{x y}^{2}+\left(\lambda_{2}-\epsilon_{y y}\right)^{2}}} .
\end{aligned}
$$

Since $\lambda_{1}$ and $\lambda_{3}$ are less than one in the cloak region, they must be replaced by dispersive quantities such as the lossless Drude model:

$$
\lambda_{i}=\epsilon_{\infty i}-\frac{\omega_{p i}^{2}}{\omega^{2}}, \quad(i=1,3),
$$

where $\omega$ is the angular frequency, $\epsilon_{\infty i}$ is the permittivity at infinite frequency, and $\omega_{p i}$ is the plasma frequency.

Using the constitutive equation $\boldsymbol{D}=\epsilon_{0} \epsilon \boldsymbol{E}$ and the decomposition $\epsilon=P \Lambda P^{T}$, we have

$$
\epsilon_{0} \boldsymbol{E}=P \Lambda^{-1} P^{T} \boldsymbol{D}
$$


which can be rewritten as

$$
\begin{aligned}
& \epsilon_{0} E_{x}=\left[\frac{1}{\lambda_{1}} p_{1}^{2}+\frac{1}{\lambda_{2}} p_{2}^{2}\right] D_{x}+\left(\frac{1}{\lambda_{2}}-\frac{1}{\lambda_{1}}\right) p_{1} p_{2} D_{y}, \\
& \epsilon_{0} E_{y}=\left(\frac{1}{\lambda_{2}}-\frac{1}{\lambda_{1}}\right) p_{1} p_{2} D_{x}+\left[\frac{1}{\lambda_{2}} p_{1}^{2}+\frac{1}{\lambda_{1}} p_{2}^{2}\right] D_{y},
\end{aligned}
$$

where $\left(D_{x}, D_{y}\right)=\boldsymbol{D}$ and $\left(E_{x}, E_{y}\right)=\boldsymbol{E}$.

Substituting $\lambda_{1}$ from (43) into (45) and simplifying the expression, we have $\left[\left(-\epsilon_{\infty 1} \omega^{2}+\omega_{p 1}^{2}\right)+\lambda_{2} \omega^{2}\right] p_{1} p_{2} D_{x}+\left[p_{1}^{2}\left(-\epsilon_{\infty 1} \omega^{2}+\omega_{p 1}^{2}\right)-\lambda_{2} p_{2}^{2} \omega^{2}\right] D_{y}=\epsilon_{0} \lambda_{2}\left(-\epsilon_{\infty 1} \omega^{2}+\omega_{p 1}^{2}\right) E_{y}$, which can be written in time domain as

$$
\begin{aligned}
& p_{1} p_{2}\left(\epsilon_{\infty 1}-\lambda_{2}\right) \frac{\partial^{2} D_{x}}{\partial t^{2}}+p_{1} p_{2} \omega_{p 1}^{2} D_{x}+\left(\epsilon_{\infty 1} p_{1}^{2}+\lambda_{2} p_{2}^{2}\right) \frac{\partial^{2} D_{y}}{\partial t^{2}}+p_{1}^{2} \omega_{p 1}^{2} D_{y} \\
& =\epsilon_{0} \lambda_{2}\left(\epsilon_{\infty 1} \frac{\partial^{2} E_{y}}{\partial t^{2}}+\omega_{p 1}^{2} E_{y}\right)
\end{aligned}
$$

where we assumed $\exp (i \omega t)$ time dependence. Similarly, changing (44) into time domain yields

$$
\begin{aligned}
& \left(\epsilon_{\infty 1} p_{2}^{2}+\lambda_{2} p_{1}^{2}\right) \frac{\partial^{2} D_{x}}{\partial t^{2}}+p_{2}^{2} \omega_{p 1}^{2} D_{x}+p_{1} p_{2}\left(\epsilon_{\infty 1}-\lambda_{2}\right) \frac{\partial^{2} D_{y}}{\partial t^{2}}+p_{1} p_{2} \omega_{p 1}^{2} D_{y} \\
& =\epsilon_{0} \lambda_{2}\left(\epsilon_{\infty 1} \frac{\partial^{2} E_{x}}{\partial t^{2}}+\omega_{p 1}^{2} E_{x}\right) .
\end{aligned}
$$

Similarly, changing the constitutive equation

$$
B=\mu_{0} \mu H=\mu_{0}\left(\epsilon_{\infty 3}-\frac{\omega_{p 3}^{2}}{\omega^{2}}\right) H
$$

into time domain, we obtain

$$
\mu_{0}\left(\epsilon_{\infty 3} \frac{\partial^{2} H}{\partial t^{2}}+\omega_{p 3}^{2} H\right)=\frac{\partial^{2} B}{\partial t^{2}}=-\nabla \times \frac{\partial \boldsymbol{E}}{\partial t},
$$

where we used (11) in the last step.

In summary, the time-domain elliptical cloaking simulation is governed by the following equations:

$$
\begin{aligned}
& \boldsymbol{D}_{t}=\nabla \times H, \\
& \epsilon_{0} \lambda_{2}\left(\epsilon_{\infty 1} \boldsymbol{E}_{t^{2}}+\omega_{p 1}^{2} \boldsymbol{E}\right)=M_{E} \boldsymbol{D}_{t^{2}}+M_{F} \boldsymbol{D}, \\
& \mu_{0}\left(\epsilon_{3} H_{t^{2}}+\omega_{p 3}^{2} H\right)=-\nabla \times \boldsymbol{E}_{t},
\end{aligned}
$$

subject to proper initial and boundary conditions. Here we denote matrices

$$
M_{E}=\left(\begin{array}{cc}
p_{1}^{2} \lambda_{2}+\epsilon_{\infty 1} p_{2}^{2} & p_{1} p_{2}\left(\epsilon_{\infty 1}-\lambda_{2}\right) \\
p_{1} p_{2}\left(\epsilon_{\infty 1}-\lambda_{2}\right) & p_{2}^{2} \lambda_{2}+\epsilon_{\infty 1} p_{1}^{2}
\end{array}\right), \quad M_{F}=\omega_{p 1}^{2}\left(\begin{array}{cc}
p_{2}^{2} & p_{1} p_{2} \\
p_{1} p_{2} & p_{1}^{2}
\end{array}\right) .
$$

The well-posedness of the model (49)-(51) can be proved similarly as we did for the cylindrical cloaking model by letting $\gamma=0$, and replacing $M_{A}$ by $M_{E}$ and $M_{B}$ by $M_{F}$. It is easy to check that all those properties of $M_{A}$ and $M_{B}$ are inherited by $M_{E}$ and $M_{F}$, respectively. For example, for any nonzero vector $(u, v)^{\prime}$, we have

$$
\begin{aligned}
(u, v) M_{E}\left(\begin{array}{c}
u \\
v
\end{array}\right) & =\left(p_{1}^{2} \lambda_{2}+\epsilon_{\infty 1} p_{2}^{2}\right) u^{2}+2 p_{1} p_{2}\left(\epsilon_{\infty 1}-\lambda_{2}\right) u v+\left(p_{2}^{2} \lambda_{2}+\epsilon_{\infty 1} p_{1}^{2}\right) v^{2} \\
& =\lambda_{2}\left(p_{1} u-p_{2} v\right)^{2}+\epsilon_{\infty 1}\left(p_{2} u+p_{1} v\right)^{2}>0
\end{aligned}
$$



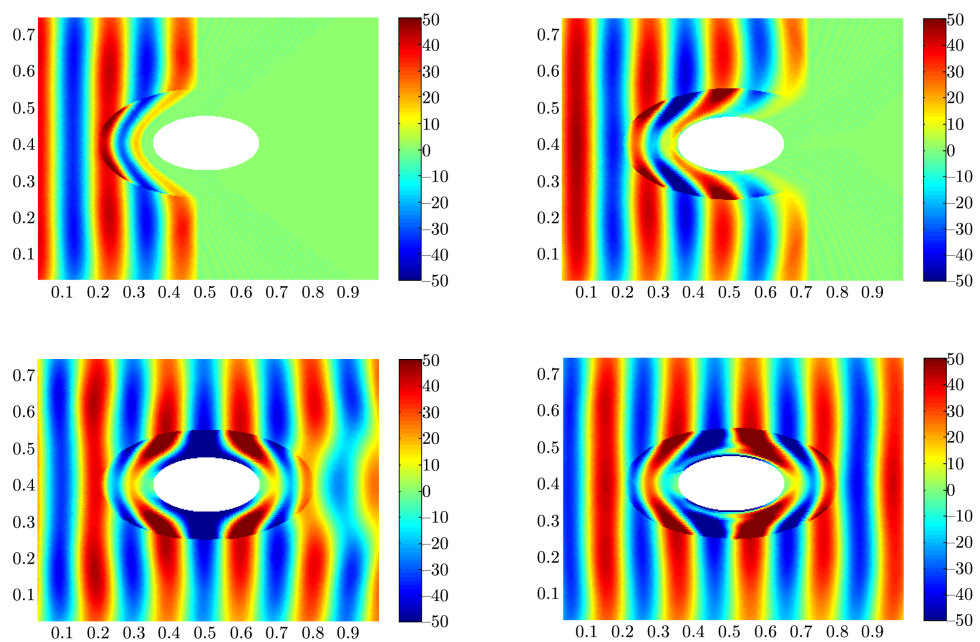

Figure 5. The electric fields $E_{y}$ at various time steps: (Top left) 4,000; (Top right) 6,000; (Bottom left) 12,000; (Bottom right) 20,000 .

and

$$
(u, v) M_{F}\left(\begin{array}{c}
u \\
v
\end{array}\right)=\omega_{p 1}^{2}\left(p_{2} u+p_{1} v\right)^{2} \geq 0 .
$$

5.2. Numerical results. Similar to the cylindrical cloak, we now construct a leapfrog type scheme for the elliptical cloak: for $n=1,2, \ldots$, find $\boldsymbol{D}_{h}^{n+\frac{1}{2}}, \boldsymbol{E}_{h}^{n+\frac{1}{2}} \in V_{h}^{0}$, $H_{h}^{n} \in U_{h}$ such that

$$
\begin{aligned}
& \quad\left(\delta_{\tau} D_{h}^{n+\frac{1}{2}}, \phi_{h}\right)=\left(H_{h}^{n}, \nabla \times \phi_{h}\right) \\
& \epsilon_{0}\left(\lambda_{2} \epsilon_{\infty 1} \delta_{\tau}^{2} \boldsymbol{E}_{h}^{n+\frac{1}{2}}, \varphi_{h}\right)+\epsilon_{0}\left(\lambda_{2} \omega_{p 1}^{2} \hat{\boldsymbol{E}}_{h}^{n}, \varphi_{h}\right) \\
& \quad=\left(M_{E} \delta_{\tau}^{2} \boldsymbol{D}_{h}^{n+\frac{1}{2}}, \varphi_{h}\right)+\left(M_{F} \hat{\boldsymbol{D}}_{h}^{n}, \varphi_{h}\right) \\
& \quad \mu_{0}\left(\epsilon_{\infty 3}\left(\delta_{\tau}^{2} H_{h}^{n+1}, \psi_{h}\right)+\left(\omega_{p 3}^{2} \hat{H}_{h}^{n+\frac{1}{2}}, \psi_{h}\right)\right)=-\left(\nabla \times \check{\boldsymbol{E}}_{h}^{n+\frac{1}{2}}, \psi_{h}\right)
\end{aligned}
$$

hold true for any $\phi_{h}, \varphi_{h} \in V_{h}^{0}, \psi_{h} \in U_{h}$.

For our elliptical cloaking simulation, we choose $a=0.075, b=0.15, k=2$, the operating frequency $f=1.5 \mathrm{GHz}$, and $\epsilon_{\infty 1}=\epsilon_{\infty 3}=1$. The elliptical cloak is embedded inside the physical domain $\Omega=[0,1]^{2}$, which is partitioned by a regular triangular mesh. An exemplary coarse mesh is shown in Figure 4 The mesh used for our simulation is obtained by uniformly refining this coarse mesh three times, and surrounded by a PML region with 12 rectangular cells of $h=0.00625$ in each direction. The total numbers of triangular elements and rectangular elements are 64896 and 8400 , respectively. The time step $\tau=4 \cdot 10^{-13}$ s is used. The incident plane wave is given by $H_{z}=0.1 \sin (\omega t)$. The electric fields $E_{y}$ obtained at different time steps are presented in Figure 5, which shows the cloaking phenomenon clearly. 


\section{Conclusions}

In this paper, we provide the well-posedness study of a 2-D cylindrical cloak modeling equations. Also, we propose a new mixed finite element scheme for simulating the cylindrical cloak. Numerical results showing the cloaking phenomena with cylindrical cloak are presented. Finally, we extend the well-posedness study to an elliptical cloak model. Numerical simulations of the elliptical cloak are also performed. Future works include extension of the proposed approach in this paper to the 3-D spherical cloak model given in [46].

\section{ACKNOWLEDGMENTS}

The authors would like to thank two anonymous referees for their insightful comments that improved our paper.

\section{References}

[1] A. Alú and N. Engheta, Achieving transparency with plasmonic and metamaterial coatings, Phys. Rev. E 72 (2005) 016623.

[2] Habib Ammari, Giulio Ciraolo, Hyeonbae Kang, Hyundae Lee, and Graeme W. Milton, Spectral theory of a Neumann-Poincaré-type operator and analysis of cloaking due to anomalous localized resonance, Arch. Ration. Mech. Anal. 208 (2013), no. 2, 667-692, DOI 10.1007/s00205-012-0605-5. MR3035988

[3] Habib Ammari, Josselin Garnier, Vincent Jugnon, Hyeonbae Kang, Hyundae Lee, and Mikyoung Lim, Enhancement of near-cloaking. Part III: Numerical simulations, statistical stability, and related questions, Multi-scale and high-contrast PDE: from modelling, to mathematical analysis, to inversion, Contemp. Math., vol. 577, Amer. Math. Soc., Providence, RI, 2012, pp. 1-24, DOI 10.1090/conm/577/11460. MR2985063

[4] Habib Ammari, Hyeonbae Kang, Hyundae Lee, and Mikyoung Lim, Enhancement of near cloaking using generalized polarization tensors vanishing structures. Part I: The conductivity problem, Comm. Math. Phys. 317 (2013), no. 1, 253-266, DOI 10.1007/s00220-012-1615-8. MR 3010374

[5] Habib Ammari, Hyeonbae Kang, Hyundae Lee, and Mikyoung Lim, Enhancement of nearcloaking. Part II: The Helmholtz equation, Comm. Math. Phys. 317 (2013), no. 2, 485-502, DOI 10.1007/s00220-012-1620-y. MR3010192

[6] Habib Ammari, Hyeonbae Kang, Hyundae Lee, Mikyoung Lim, and Sanghyeon Yu, Enhancement of near cloaking for the full Maxwell equations, SIAM J. Appl. Math. 73 (2013), no. 6, 2055-2076, DOI 10.1137/120903610. MR3127003

[7] Gang Bao, Peijun Li, and Haijun Wu, An adaptive edge element method with perfectly matched absorbing layers for wave scattering by biperiodic structures, Math. Comp. 79 (2010), no. 269, 1-34, DOI 10.1090/S0025-5718-09-02257-1. MR2552215 (2011a:65427)

[8] Rudi Beck, Ralf Hiptmair, Ronald H. W. Hoppe, and Barbara Wohlmuth, Residual based a posteriori error estimators for eddy current computation (English, with English and French summaries), M2AN Math. Model. Numer. Anal. 34 (2000), no. 1, 159-182, DOI 10.1051/m2an:2000136. MR1735971 (2000k:65203)

[9] Jean-Pierre Berenger, A perfectly matched layer for the absorption of electromagnetic waves, J. Comput. Phys. 114 (1994), no. 2, 185-200, DOI 10.1006/jcph.1994.1159. MR1294924 (95e:78002)

[10] S. C. Brenner, J. Cui, Z. Nan, and L.-Y. Sung, Hodge decomposition for divergence-free vector fields and two-dimensional Maxwell's equations, Math. Comp. 81 (2012), no. 278, 643-659, DOI 10.1090/S0025-5718-2011-02540-8. MR2869031

[11] Susanne C. Brenner, Fengyan Li, and Li-Yeng Sung, A locally divergence-free nonconforming finite element method for the time-harmonic Maxwell equations, Math. Comp. 76 (2007), no. 258, 573-595, DOI 10.1090/S0025-5718-06-01950-8. MR2291828(2008c:65316)

[12] H. Chen, C.T. Chan and P. Sheng, Transformation optics and metamaterials, Nature Materials 9 (2010) 387-396.

[13] Zhiming Chen, Qiang Du, and Jun Zou, Finite element methods with matching and nonmatching meshes for Maxwell equations with discontinuous coefficients, SIAM J. Numer. Anal. 37 (2000), no. 5, 1542-1570, DOI 10.1137/S0036142998349977. MR 1759906 (2001h:78044) 
[14] Eric T. Chung, Patrick Ciarlet Jr., and Tang Fei Yu, Convergence and superconvergence of staggered discontinuous Galerkin methods for the three-dimensional Maxwell's equations on Cartesian grids, J. Comput. Phys. 235 (2013), 14-31, DOI 10.1016/j.jcp.2012.10.019. MR 3017583

[15] P. Ciarlet Jr. and Jun Zou, Fully discrete finite element approaches for time-dependent Maxwell's equations (English, with English and French summaries), Numer. Math. 82 (1999), no. 2, 193-219, DOI 10.1007/s002110050417. MR.1685459 (2000c:65083)

[16] S.A. Cummer, B.-I. Popa, D. Schurig, D.R. Smith and J. Pendry, Full-wave simulations of electromagnetic cloaking structures, Phys. Rev. E 74 (2006) 036621.

[17] Leszek Demkowicz, Jason Kurtz, David Pardo, Maciej Paszyński, Waldemar Rachowicz, and Adam Zdunek, Computing with hp-Adaptive Finite Elements. Vol. 2, Chapman \& Hall/CRC Applied Mathematics and Nonlinear Science Series, Chapman \& Hall/CRC, Boca Raton, FL, 2008. Frontiers: three dimensional elliptic and Maxwell problems with applications. MR2406401(2009e:65172)

[18] Paolo Fernandes and Mirco Raffetto, Well-posedness and finite element approximability of time-harmonic electromagnetic boundary value problems involving bianisotropic materials and metamaterials, Math. Models Methods Appl. Sci. 19 (2009), no. 12, 2299-2335, DOI 10.1142/S0218202509004121. MR2599662 (2011b:65222)

[19] M. Fridman, A. Farsi, Y. Okawachi and A.L. Gaeta, Demonstration of temporal cloaking, Nature 481 (2012) 62-65.

[20] Allan Greenleaf, Matti Lassas, and Gunther Uhlmann, On nonuniqueness for Calderón's inverse problem, Math. Res. Lett. 10 (2003), no. 5-6, 685-693, DOI 10.4310/MRL.2003.v10.n5.a11. MR2024725(2005f:35316)

[21] Allan Greenleaf, Yaroslav Kurylev, Matti Lassas, and Gunther Uhlmann, Cloaking devices, electromagnetic wormholes, and transformation optics, SIAM Rev. 51 (2009), no. 1, 3-33, DOI 10.1137/080716827. MR2481110(2010b:35484)

[22] S. Guenneau, R.C. McPhedran, S. Enoch, A.B. Movchan, M. Farhat and N.-A. P. Nicorovici, The colours of cloaks, J. Opt. 13 (2011) 024014.

[23] F. Guevara Vasquez, G.W. Milton and D. Onofrei, Broadband exterior cloaking, Opt. Express 17 (2009) 14800-14805.

[24] J. Hao, W. Yan and M. Qiu, Super-reflection and cloaking based on zero index metamaterial, Appl. Phys. Lett. 96, 101109 (2010).

[25] Y. Hao and R. Mittra, FDTD Modeling of Metamaterials: Theory and Applications, Artech House Publishers, 2008.

[26] Jan S. Hesthaven and Tim Warburton, Nodal Discontinuous Galerkin Methods: Algorithms, analysis, and applications, Texts in Applied Mathematics, vol. 54, Springer, New York, 2008. MR2372235(2008k:65002)

[27] Paul Houston, Ilaria Perugia, Anna Schneebeli, and Dominik Schötzau, Interior penalty method for the indefinite time-harmonic Maxwell equations, Numer. Math. 100 (2005), no. 3, 485-518, DOI 10.1007/s00211-005-0604-7. MR2194528(2006k:65323)

[28] W.X. Jiang, T.J. Cui, G.X. Yu, X.Q. Lin, Q. Cheng, J.Y. Chin, Arbitrarily ellipticalcylindrical invisible cloaking, J. Phys. D: Appl. Phys. 41 (2008), 085504.

[29] Robert V. Kohn, Daniel Onofrei, Michael S. Vogelius, and Michael I. Weinstein, Cloaking via change of variables for the Helmholtz equation, Comm. Pure Appl. Math. 63 (2010), no. 8, 973-1016, DOI 10.1002/cpa.20326. MR2642383 (2011j:78004)

[30] R. V. Kohn, H. Shen, M. S. Vogelius, and M. I. Weinstein, Cloaking via change of variables in electric impedance tomography, Inverse Problems 24 (2008), no. 1, 015016, 21, DOI 10.1088/0266-5611/24/1/015016. MR2384775(2008m:78014)

[31] Ulf Leonhardt, Optical conformal mapping, Science 312 (2006), no. 5781, 1777-1780, DOI 10.1126/science.1126493. MR2237569

[32] Ulf Leonhardt and Thomas Philbin, Geometry and Light: The Science of Invisibility, Dover Publications Inc., Mineola, NY, 2010. MR2798945(2012f:53001)

[33] U. Leonhardt and T. Tyc, Broadband invisibility by non-Euclidean cloaking, Science 323 (2009) 110-112.

[34] Jichun Li and Yunqing Huang, Mathematical simulation of cloaking metamaterial structures, Adv. Appl. Math. Mech. 4 (2012), no. 1, 93-101. MR2876653 (2012m:78015) 
[35] Jichun Li and Yunqing Huang, Time-domain Finite Element Methods for Maxwell's Equations in Metamaterials, Springer Series in Computational Mathematics, vol. 43, Springer, Heidelberg, 2013. MR.3013583

[36] Jichun Li, Yunqing Huang, and Wei Yang, Developing a time-domain finite-element method for modeling of electromagnetic cylindrical cloaks, J. Comput. Phys. 231 (2012), no. 7, 28802891, DOI 10.1016/j.jcp.2011.12.026. MR2882105

[37] Jingzhi Li, Hongyu Liu, and Hongpeng Sun, Enhanced approximate cloaking by SH and FSH lining, Inverse Problems 28 (2012), no. 7, 075011, 21, DOI 10.1088/0266-5611/28/7/075011. MR2946799

[38] Z. Liang, P. Yao, X. Sun and X. Jiang, The physical picture and the essential elements of the dynamical process for dispersive cloaking structures, Appl. Phys. Lett. 92, 131118 (2008).

[39] Hongyu Liu and Ting Zhou, On approximate electromagnetic cloaking by transformation media, SIAM J. Appl. Math. 71 (2011), no. 1, 218-241, DOI 10.1137/10081112X. MR2776835 (2012f:78005)

[40] R. Liu, C. Ji, J.J. Mock, J.Y. Chin, T.J. Cui and D.R. Smith, Broadband ground-plane cloak, Science 323 (2009) 366-369.

[41] Graeme W. Milton and Nicolae-Alexandru P. Nicorovici, On the cloaking effects associated with anomalous localized resonance, Proc. R. Soc. Lond. Ser. A Math. Phys. Eng. Sci. 462 (2006), no. 2074, 3027-3059, DOI 10.1098/rspa.2006.1715. MR2263683 (2008e:78018)

[42] Peter Monk, Finite Element Methods for Maxwell's Equations, Numerical Mathematics and Scientific Computation, Oxford University Press, New York, 2003. MR2059447|(2005d:65003)

[43] Hoai-Minh Nguyen, Approximate cloaking for the Helmholtz equation via transformation optics and consequences for perfect cloaking, Comm. Pure Appl. Math. 65 (2012), no. 2, 155-186, DOI 10.1002/cpa.20392. MR2855543

[44] V.C. Nguyen, L. Chen and K. Halterman, Total transmission and total reflection by zero index metamaterials with defects, Phys. Rev. Lett. 105, 233908 (2010).

[45] N. Okada and J.B. Cole, FDTD modeling of a cloak with a nondiagonal permittivity tensor, ISRN Optics, Article ID 536209, doi:10.5402/2012/536209, 2012.

[46] J. B. Pendry, D. Schurig, and D. R. Smith, Controlling electromagnetic fields, Science 312 (2006), no. 5781, 1780-1782, DOI 10.1126/science.1125907. MR2237570

[47] C. Scheid and S. Lanteri, Convergence of a Discontinuous Galerkin scheme for the mixed time domain Maxwell's equations in dispersive media, IMA J. Numer. Anal. (in press). doi:10.1093/imanum/drs008.

[48] D. Schurig, J. J. Mock, B. J. Justice, S. A. Cummer, J. B. Pendry, A. F. Starr, D. R. Smith, Metamaterial electromagnetic cloak at microwave frequencies, Science 314 (2006) 977-980.

[49] Simon Shaw, Finite element approximation of Maxwell's equations with Debye memory, Adv. Numer. Anal. (2010), Art. ID 923832, 28. MR2747091 (2012c:65207)

[50] Bo Wang, Ziqing Xie, and Zhimin Zhang, Error analysis of a discontinuous Galerkin method for Maxwell equations in dispersive media, J. Comput. Phys. 229 (2010), no. 22, 8552-8563, DOI 10.1016/j.jcp.2010.07.038. MR2719188(2011f:78011)

[51] M. Yan, W. Yan and M. Qiu, Invisibility cloaking by coordinate transformation, Progress in Optics 52 (2009) 261-304.

[52] Y. Zhao, C. Argyropoulos and Y. Hao, Full-wave finite-difference time-domain simulation of electromagnetic cloaking structures, Optics Express 16 (2008) 6717-6730.

[53] Liuqiang Zhong, Long Chen, Shi Shu, Gabriel Wittum, and Jinchao Xu, Convergence and optimality of adaptive edge finite element methods for time-harmonic Maxwell equations, Math. Comp. 81 (2012), no. 278, 623-642, DOI 10.1090/S0025-5718-2011-02544-5. MR2869030

Department of Mathematical Sciences, University of Nevada Las Vegas, Las Vegas, NEVADA 89154-4020

E-mail address: jichun@unlv.nevada.edu

Hunan Key Laboratory for Computation and Simulation in Science and Engineering,

Xiangtan University, Xiangtan 411105, China

E-mail address: huangyq@xtu.edu.cn

Hunan Key Laboratory for Computation and Simulation in Science and Engineering,

Xiangtan University, Xiangtan 411105, China

E-mail address: yangweixtu@126.com 